\title{
390.
}

\section{THEOREM RELATING TO THE FOUR CONICS WHICH TOUCH THE SAME TWO LINES AND PASS THROUGH THE SAME FOUR POINTS.}

[From the Quarterly Journal of Pure and Applied Mathematics, vol. viII. (1867), pp. 162-167.]

The sides of the triangle formed by the given points meet one of the given lines in three points, say $P, Q, R$; and on this same line we have four points of contact, say $A_{1}, A_{2}, A_{3}, A_{4}$; any two pairs, say $A_{1}, A_{2} ; A_{3}, A_{4}$, form with a properly selected pair, say $Q, R$, out of the above-mentioned three points, an involution; and we have thus the three involutions

$$
\begin{aligned}
& \left(A_{1}, A_{2} ; A_{3}, A_{4} ; \quad Q, R\right), \\
& \left(A_{1}, A_{3} ; A_{4}, A_{2} ; R, P\right), \\
& \left(A_{1}, A_{4} ; A_{2}, A_{3} ; P, Q\right) .
\end{aligned}
$$

To prove this, let $x=0, y=0$ be the equations of the given lines, and take for the equations of the sides of the triangle formed by the given points

$$
\begin{aligned}
& b x+a y-a b=0, \\
& b^{\prime} x+a^{\prime} y-a^{\prime} b^{\prime}=0, \\
& b^{\prime \prime} x+a^{\prime \prime} y-a^{\prime \prime} b^{\prime \prime}=0
\end{aligned}
$$

the equation of any one of the four conics may be written

$$
\frac{L a b}{3 x+a y-a b}+\frac{L^{\prime} a^{\prime} b^{\prime}}{b^{\prime} x+a^{\prime} y-a^{\prime} b^{\prime}}+\frac{L^{\prime \prime} a^{\prime \prime} b^{\prime \prime}}{b^{\prime \prime} x+a^{\prime \prime} y-a^{\prime \prime} b^{\prime \prime}}=0,
$$

and if this touches the axis of $x$, say at the point $x=\alpha$, then we must have

$$
\frac{L a}{x-a}+\frac{L^{\prime} a^{\prime}}{x-a^{\prime}}+\frac{L^{\prime \prime} a^{\prime \prime}}{x-a^{\prime \prime}}=\frac{-K(x-a)^{2}}{(x-a)\left(x-a^{\prime}\right)\left(x-a^{\prime \prime}\right)}
$$


or, assuming as we may do, $K=-\left(a^{\prime}-a^{\prime \prime}\right)\left(a^{\prime \prime}-a\right)\left(a-a^{\prime}\right)$, this gives

$$
\begin{aligned}
& L a=(a-a)^{2}\left(a^{\prime}-a^{\prime \prime}\right), \\
& L^{\prime} a^{\prime}=\left(a^{\prime}-\alpha\right)^{2}\left(a^{\prime \prime}-a\right), \\
& L^{\prime \prime} a^{\prime \prime}=\left(a^{\prime \prime}-\alpha\right)^{2}\left(a-a^{\prime}\right) .
\end{aligned}
$$

But in the same manner, if the conic touch the axis of $y$, say at the point $y=\beta$, we have

$$
\begin{aligned}
& L b=(b-\beta)^{2}\left(b^{\prime}-b^{\prime \prime}\right), \\
& L^{\prime} b^{\prime}=\left(b^{\prime}-\beta\right)^{2}\left(b^{\prime \prime}-b\right) \\
& L^{\prime \prime} b^{\prime \prime}=\left(b^{\prime \prime}-\beta\right)^{2}\left(b-b^{\prime}\right)
\end{aligned}
$$

and thence

$$
\begin{aligned}
& b(a-\alpha)^{2}\left(a^{\prime}-a^{\prime \prime}\right): b^{\prime}\left(a^{\prime}-\alpha\right)^{2}\left(a^{\prime \prime}-a\right): b^{\prime \prime}\left(a^{\prime \prime}-\alpha\right)^{2}\left(a-a^{\prime}\right) \\
= & a(b-\beta)^{2}\left(b^{\prime}-b^{\prime \prime}\right): a^{\prime}\left(b^{\prime}-\beta\right)^{2}\left(b^{\prime \prime}-b\right): a^{\prime \prime}\left(b^{\prime \prime}-\beta\right)^{2}\left(b-b^{\prime}\right) .
\end{aligned}
$$

Putting

$$
\begin{aligned}
& P=a b\left(a^{\prime}-a^{\prime \prime}\right)\left(b^{\prime}-b^{\prime \prime}\right) \\
& P^{\prime}=a^{\prime} b^{\prime}\left(a^{\prime \prime}-a\right)\left(b^{\prime \prime}-b\right) \\
& P^{\prime \prime}=a^{\prime \prime} b^{\prime \prime}\left(a-a^{\prime}\right)\left(b-b^{\prime}\right)
\end{aligned}
$$

we have

$(a-\alpha)^{2} \frac{P}{a^{2}}:\left(a^{\prime}-\alpha\right)^{2} \frac{P^{\prime}}{a^{\prime 2}}:\left(a^{\prime \prime}-\alpha\right)^{2} \frac{P^{\prime \prime}}{a^{\prime \prime 2}}=(b-\beta)^{2}\left(b^{\prime}-b^{\prime \prime}\right)^{2}:\left(b^{\prime}-\beta\right)^{2}\left(b^{\prime \prime}-b\right)^{2}:\left(b^{\prime \prime}-\beta\right)^{2}\left(b-b^{\prime}\right)^{2} ;$

and thence

$$
\begin{aligned}
& (a-\alpha) \frac{\sqrt{ }(P)}{a}:\left(a^{\prime}-\alpha\right) \frac{\sqrt{ }\left(P^{\prime}\right)}{a^{\prime}}-\left(a^{\prime \prime}-\alpha\right) \frac{\sqrt{ }\left(P^{\prime \prime}\right)}{a^{\prime \prime}} \\
= & (b-\beta)\left(b^{\prime}-b^{\prime \prime}\right):\left(b^{\prime}-\beta\right)\left(b^{\prime \prime}-b\right):\left(b^{\prime \prime}-\beta\right)\left(b-b^{\prime}\right),
\end{aligned}
$$

which gives

$$
(a-a) \frac{\sqrt{ }(P)}{a}+\left(a^{\prime}-\alpha\right) \frac{\sqrt{ }\left(P^{\prime}\right)}{a^{\prime}}+\left(a^{\prime \prime}-\alpha\right) \frac{\sqrt{ }\left(P^{\prime \prime}\right)}{a^{\prime \prime}}=0
$$

and we have in like manner

$$
(b-\beta) \frac{\sqrt{ }(P)}{b}+\left(b^{\prime}-\beta\right) \frac{\sqrt{ }\left(P^{\prime}\right)}{b^{\prime}}+\left(b^{\prime \prime}-\beta\right)^{\frac{\sqrt{ }\left(P^{\prime \prime}\right)}{b^{\prime \prime}}}=0
$$

but the first of these equations is alone required for the present purpose. Putting for shortness

$$
P=a^{2} X, \quad P^{\prime}=a^{\prime 2} X^{\prime}, \quad P^{\prime \prime}=a^{\prime \prime 2} X^{\prime \prime},
$$

the equation is

$$
(a-\alpha) \sqrt{ }(X)+\left(a^{\prime}-\alpha\right) \sqrt{ }\left(X^{\prime}\right)+\left(a^{\prime \prime}-\alpha\right) \sqrt{ }\left(X^{\prime \prime}\right)
$$


and by attributing the signs + and - to the radicals, we have, corresponding to the four conics, the equations

$$
\begin{array}{r}
\left(a-\alpha_{1}\right) \sqrt{ }(X)+\left(a^{\prime}-\alpha_{1}\right) \sqrt{ }\left(X^{\prime}\right)+\left(a^{\prime \prime}-\alpha_{1}\right) \sqrt{ }\left(X^{\prime \prime}\right)=0, \\
-\left(a-\alpha_{2}\right) \sqrt{ }(X)+\left(a^{\prime}-\alpha_{2}\right) \sqrt{ }\left(X^{\prime}\right)+\left(a^{\prime \prime}-\alpha_{2}\right) \sqrt{ }\left(X^{\prime \prime}\right)=0, \\
\left(a-\alpha_{3}\right) \sqrt{ }(X)-\left(a^{\prime}-\alpha_{3}\right) \sqrt{ }\left(X^{\prime}\right)+\left(a^{\prime \prime}-\alpha_{3}\right) \sqrt{ }\left(X^{\prime \prime}\right)=0, \\
\left(a-\alpha_{4}\right) \sqrt{ }(X)+\left(a^{\prime}-\alpha_{4}\right) \sqrt{ }\left(X^{\prime}\right)-\left(a^{\prime \prime}-\alpha_{4}\right) \sqrt{ }\left(X^{\prime \prime}\right)=0,
\end{array}
$$

where $\alpha_{1}, \alpha_{2}, \alpha_{3}, \alpha_{4}$ are the values of $\alpha$ for the four conics respectively.

Eliminating $a^{\prime \prime}$ we obtain the system of three equations

$$
\begin{array}{rrr}
\left(2 a-\alpha_{1}-\alpha_{2}\right) \sqrt{ }(X)+ & \left(\alpha_{2}-\alpha_{1}\right) \sqrt{ }\left(X^{\prime}\right) & +\left(\alpha_{2}-\alpha_{1}\right) \sqrt{ }\left(X^{\prime \prime}\right)=0 \\
\left(\alpha_{3}-\alpha_{1}\right) \sqrt{ }(X)+\left(2 a^{\prime}-\alpha_{1}-\alpha_{3}\right) \sqrt{ }\left(X^{\prime}\right) & +\left(\alpha_{3}-\alpha_{1}\right) \sqrt{ }\left(X^{\prime \prime}\right)=0 \\
\left(\alpha_{1}+\alpha_{2}-\alpha_{3}-\alpha_{4}\right) \sqrt{ }(X)+\left(\alpha_{1}+\alpha_{3}-\alpha_{2}-\alpha_{4}\right) \sqrt{ }\left(X^{\prime}\right)+\left(\alpha_{1}+\alpha_{4}-\alpha_{2}-\alpha_{3}\right) \sqrt{ }\left(X^{\prime \prime}\right)=0
\end{array}
$$

and then eliminating the radicals we have

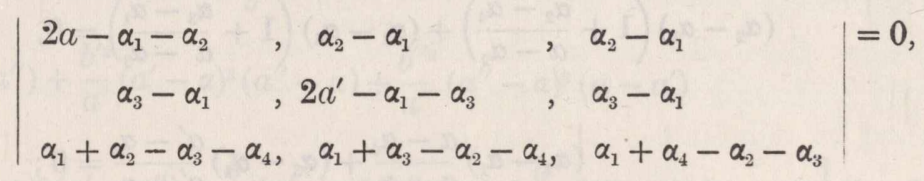

which is in fact

$$
-4\left|\begin{array}{ccc}
1, & a+a^{\prime}, & a a^{\prime} \\
1, & \alpha_{1}+\alpha_{4}, & \alpha_{1} \alpha_{4} \\
1, & \alpha_{2}+\alpha_{3}, & \alpha_{2} \alpha_{3}
\end{array}\right|=0
$$

as may be verified by actual expansion; the transformation of the determinant is a peculiar one.

The foregoing result was originally obtained as follows, viz. writing for a moment

$$
\begin{array}{r}
a \sqrt{ }(X)+a^{\prime} \sqrt{ }\left(X^{\prime}\right)+a^{\prime \prime} \sqrt{ }\left(X^{\prime \prime}\right)=\Theta, \\
\sqrt{ }(X)+\sqrt{ }\left(X^{\prime}\right)+\sqrt{ }\left(X^{\prime \prime}\right)=\Phi,
\end{array}
$$

the four equations are

$$
\begin{aligned}
& \Theta-\alpha_{1} \Phi=0 \\
& \Theta-\alpha_{2} \Phi=2\left(a-\alpha_{2}\right) \sqrt{ }(X), \\
& \Theta-\alpha_{3} \Phi=2\left(a^{\prime}-\alpha_{3}\right) \sqrt{ }\left(X^{\prime}\right) \\
& \Theta-\alpha_{4} \Phi=2\left(a^{\prime \prime}-\alpha_{4}\right) \sqrt{ }\left(X^{\prime \prime}\right)
\end{aligned}
$$

these give

$$
\begin{aligned}
& \left(\alpha_{1}-\alpha_{2}\right) \Phi=2\left(a-\alpha_{2}\right) \sqrt{ }(X), \\
& \left(\alpha_{1}-\alpha_{3}\right) \Phi=2\left(a^{\prime}-\alpha_{3}\right) \sqrt{ }\left(X^{\prime}\right) \\
& \left(\alpha_{1}-\alpha_{4}\right) \Phi=2\left(a^{\prime \prime}-\alpha_{4}\right) \sqrt{ }\left(X^{\prime \prime}\right) .
\end{aligned}
$$


From the last equation we have

that is

$$
\begin{aligned}
\left(\alpha_{1}-\alpha_{4}\right) \Phi & =2\left\{\Theta-a \sqrt{ }(X)-a^{\prime} \sqrt{ }\left(X^{\prime}\right)\right\}-2 \alpha_{4}\left\{\Phi-\sqrt{ }(X)-\sqrt{ }\left(X^{\prime}\right)\right\} \\
& =2\left(\alpha_{1}-\alpha_{4}\right) \Phi-2\left(a-\alpha_{4}\right) \sqrt{ }(X)-2\left(a^{\prime}-\alpha_{4}\right) \sqrt{ }\left(X^{\prime}\right)
\end{aligned}
$$

$$
\left(\alpha_{1}-\alpha_{4}\right) \Phi-2\left(a-\alpha_{4}\right) \sqrt{ }(X)-2\left(a^{\prime}-\alpha_{4}\right) \sqrt{ }\left(X^{\prime}\right)=0 ;
$$

or substituting for $\sqrt{ }(X), \sqrt{ }\left(X^{\prime}\right)$ their values in terms of $\Phi$, we find

$$
\alpha_{1}-\alpha_{4}-\frac{\left(a-\alpha_{4}\right)\left(\alpha_{1}-\alpha_{2}\right)}{a-\alpha_{2}}-\frac{\left(a^{\prime}-\alpha_{4}\right)\left(\alpha_{1}-\alpha_{3}\right)}{a^{\prime}-\alpha_{3}}=0,
$$

which may be written

that is

$$
\alpha_{1}-\alpha_{4}-\left(\alpha_{1}-\alpha_{2}\right)\left(1+\frac{\alpha_{2}-\alpha_{4}}{a-\alpha_{2}}\right)-\left(\alpha_{1}-\alpha_{3}\right)\left(1+\frac{\alpha_{3}-\alpha_{4}}{\alpha^{\prime}-\alpha_{3}}\right)=0
$$

$$
\alpha_{2}+\alpha_{3}-\alpha_{1}-\alpha_{4}+\frac{\left(\alpha_{2}-\alpha_{1}\right)\left(\alpha_{2}-\alpha_{4}\right)}{a-\alpha_{2}}+\frac{\left(\alpha_{3}-\alpha_{1}\right)\left(\alpha_{3}-\alpha_{4}\right)}{a^{\prime}-\alpha_{3}}=0
$$

or again

$$
\left(\alpha_{2}-\alpha_{1}\right)\left(1+\frac{\alpha_{2}-\alpha_{4}}{a-\alpha_{2}}\right)+\left(\alpha_{3}-\alpha_{4}\right)\left(1+\frac{\alpha_{3}-\alpha_{1}}{a^{\prime}-\alpha_{3}}\right)=0
$$

that is

or finally

$$
\left(\alpha_{2}-\alpha_{1}\right) \frac{a-\alpha_{4}}{a-\alpha_{2}}+\left(\alpha_{3}-\alpha_{4}\right) \frac{a^{\prime}-\alpha_{1}}{a^{\prime}-\alpha_{3}}=0
$$

$$
\left(\alpha_{2}-\alpha_{1}\right)\left(a-\alpha_{4}\right)\left(a^{\prime}-\alpha_{3}\right)+\left(\alpha_{3}-\alpha_{4}\right)\left(a-\alpha_{2}\right)\left(a^{\prime}-\alpha_{1}\right)=0,
$$

which is a known form of the relation

$$
\left|\begin{array}{lll}
1, & a+a^{\prime}, & a a^{\prime} \\
1, & \alpha_{1}+\alpha_{4}, & \alpha_{1} \alpha_{4} \\
1, & \alpha_{2}+\alpha_{3}, & \alpha_{2} \alpha_{3}
\end{array}\right|=0
$$

which gives the involution of the quantities $a, a^{\prime} ; \alpha_{1}, \alpha_{4} ; \alpha_{2}, \alpha$.

We have in like manner

$$
\left|\begin{array}{ccc}
1, & a^{\prime}+a^{\prime \prime}, & a^{\prime} a^{\prime \prime} \\
1, & \alpha_{1}+\alpha_{2}, & \alpha_{1} \alpha_{2} \\
1, & \alpha_{3}+\alpha_{4}, & \alpha_{3} \alpha_{4}
\end{array}\right|=0
$$

and

$$
\left|\begin{array}{ccc}
1, & a^{\prime \prime}+a, & a^{\prime \prime} a \\
1, & \alpha_{1}+\alpha_{3}, & \alpha_{1} \alpha_{3} \\
1, & \alpha_{2}+\alpha_{4}, & \alpha_{2} \alpha_{4}
\end{array}\right|=0
$$

which give the involutions of the systems $a^{\prime}, a^{\prime \prime} ; \alpha_{1}, \alpha_{2} ; \alpha_{3}, \alpha_{4}$ and $a^{\prime \prime}, a ; \alpha_{1}, \alpha_{3} ; \alpha_{2}, \alpha_{4}$ respectively. 
It may be remarked that the equation of the conic passing through the three points and touching the axis of $x$ in the point $x=\alpha$ is

$$
\frac{(a-a)^{2}\left(a^{\prime}-a^{\prime \prime}\right) b}{b x+a y-a b}+\frac{\left(a^{\prime}-\alpha\right)^{2}\left(a^{\prime \prime}-a\right) b^{\prime}}{b^{\prime} x+a^{\prime} y-a^{\prime} b^{\prime}}+\frac{\left(a^{\prime \prime}-\alpha\right)^{2}\left(a-a^{\prime}\right) b^{\prime \prime}}{b^{\prime \prime} x+a^{\prime \prime} y-a^{\prime \prime} b^{\prime \prime}}=0,
$$

and when this meets the axis of $y$ we have

$$
\frac{\frac{b}{a}(a-\alpha)^{2}\left(a^{\prime}-a^{\prime \prime}\right)}{y-b}+\frac{\frac{b^{\prime}}{a^{\prime}}\left(a^{\prime}-\alpha\right)^{2}\left(a^{\prime \prime}-a\right)}{y-b^{\prime}}+\frac{\frac{b^{\prime \prime}}{a^{\prime \prime}}\left(a^{\prime \prime}-\alpha\right)^{2}\left(a-a^{\prime}\right)}{y-b^{\prime \prime}}=0 .
$$

Hence, if this touches the axis of $y$ in the point $y=\beta$, the left-hand side must be

$$
=\frac{\left[\frac{b}{a}(a-\alpha)^{2}\left(a^{\prime}-a^{\prime \prime}\right)+\frac{b^{\prime}}{a^{\prime}}\left(a^{\prime}-\alpha\right)^{2}\left(a^{\prime \prime}-a\right)+\frac{b^{\prime \prime}}{a^{\prime \prime}}\left(a^{\prime \prime}-\alpha\right)^{2}\left(a-a^{\prime}\right)\right](y-\beta)^{2}}{(y-b)\left(y-b^{\prime}\right)\left(y-b^{\prime \prime}\right)},
$$

and equating the coefficients of $\frac{1}{y^{2}}$, we have

$$
\begin{aligned}
& \frac{b^{2}}{a}(a-\alpha)^{2}\left(a^{\prime}-a^{\prime \prime}\right)+\frac{b^{\prime 2}}{a^{\prime}}\left(a^{\prime}-\alpha\right)^{2}\left(a^{\prime \prime}-a\right)+\frac{b^{\prime \prime 2}}{a^{\prime \prime}}\left(a^{\prime \prime}-\alpha\right)^{2}\left(a-a^{\prime}\right) \\
& \quad=\left[\frac{b}{a}(a-\alpha)^{2}\left(a^{\prime}-a^{\prime \prime}\right)+\frac{b^{\prime}}{a^{\prime}}\left(a^{\prime}-\alpha\right)^{2}\left(a^{\prime \prime}-a\right)+\frac{b^{\prime \prime}}{a^{\prime \prime}}\left(a^{\prime \prime}-\alpha\right)^{2}\left(a-a^{\prime}\right)\right]\left(b+b^{\prime}+b^{\prime \prime}-2 \beta\right),
\end{aligned}
$$

or what is the same thing,

$$
\begin{aligned}
& \frac{b\left(b^{\prime}+b^{\prime \prime}\right)}{a}(a-\alpha)^{2}\left(a^{\prime}-a^{\prime \prime}\right)+\frac{b^{\prime}\left(b^{\prime \prime}+b\right)}{a^{\prime}}\left(a^{\prime}-\alpha\right)^{2}\left(a^{\prime \prime}-a\right)+\frac{b^{\prime \prime}\left(b+b^{\prime}\right)}{a^{\prime \prime}}\left(a^{\prime \prime}-\alpha\right)^{2}\left(a-a^{\prime}\right) \\
& \quad=2 \beta\left[\frac{b}{a}(a-\alpha)^{2}\left(a^{\prime}-a^{\prime \prime}\right)+\frac{b^{\prime}}{a^{\prime}}\left(a^{\prime}-\alpha\right)^{2}\left(a^{\prime \prime}-a\right)+\frac{b^{\prime \prime}}{a^{\prime \prime}}\left(a^{\prime \prime}-\alpha\right)^{2}\left(a-a^{\prime}\right)\right],
\end{aligned}
$$

which gives $\beta$ in terms of $\alpha$, that is $\beta_{1}, \beta_{2}, \beta_{3}, \beta_{4}$ in terms of $\alpha_{1}, \alpha_{2}, \alpha_{3}, \alpha_{4}$ respectively.

Cambridge, 30 November, 1863. 